VOL. 67 (2003) [177-185]

\title{
THE EFFECT OF THE DUAL ON A BANACH SPACE AND THE WEAK FIXED POINT PROPERTY
}

\author{
Tim Dalby
}

\begin{abstract}
A number of Banach space properties have been shown to imply the weak fixed point property. If the dual of a Banach space were to possess some of these properties then the original space can been shown to satisfy related conditions.
\end{abstract}

\section{INTRODUCTION}

A Banach space is said to have the weak fixed point property if every nonexpansive mapping on every nonempty weak compact convex set has a fixed point. The usual approach to show that a certain property is sufficient for the weak fixed point property is to assume that $X$ does not satisfy the weak fixed point property and then try for a contradiction. Failure to have the weak fixed point property means that there is a nonempty weak compact convex set $C$ and a fixed point free nonexpansive mapping $T: C \rightarrow C$. Thus $C$ cannot be a singleton, so $\operatorname{diam}(C)>0$. From here it is possible show the existence of an approximate fixed point sequence, $\left(x_{n}\right)$; in $C$. That is, a sequence that satisfies

$$
\lim _{n}\left\|T x_{n}-x_{n}\right\|=0 \text {. }
$$

An important step in many proofs is the introduction of minimal invariant subsets. If $K$ is a minimal invariant subset of $C$ for $T$ then $K$ is nonempty weak compact, convex and $T(K) \subset K$. Moreover, $K$ has no nonempty weak compact convex proper subsets that are $T$-invariant. The existence of such minimal subsets comes from an application of Zorn's lemma.

Goebel [6] and Karlovitz [8] produced the following result. If $K$ is a minimal invariant subset and $\left(x_{n}\right)$ is an approximate fixed point sequence in $K$ then

$$
\lim _{n}\left\|x-x_{n}\right\|=\operatorname{diam}(D) \text { for all } x \in K .
$$

This is commonly known as the Goebel-Karlovitz Lemma.

Received 19th February, 2002

The author is grateful to the referee for pointing out the work of Takahashi and Kato [17] and GarcíaFalset, Llorens-Fuster and Mazcuñan-Navarro [5].

Copyright Clearance Centre, Inc. Serial-fee code: 0004-9727/03 \$A2.00+0.00. 
Starting with an approximate fixed point sequence, $\left(x_{n}\right)$ in $K$, we may assume that $\left(x_{n}\right)$ is weak convergent and by a dilation and a translation we can further assume that $x_{n} \rightarrow 0$ and $\operatorname{diam}(K)=1$. So $0 \in K$ and $\lim _{n}\left\|x_{n}\right\|=1$. This situation amounts to the usual set up in the start of many proofs of the weak fixed point property.

In addition, it may be assumed that $X$ is separable, see for example Goebel and Kirk [7]. So throughout this paper $X$ will be assumed to be an infinite dimensional separable Banach space. Note that one consequence of the assumption that $X$ is separable is that the dual unit ball, $B_{X^{*}}$, is weak* sequentially compact. Thus in a number of instances, sequences in $B_{X}$. will be assumed, without loss of generality, to be weak* convergent.

\section{Preliminaries}

In the numerous attempts to find sufficient conditions for the weak fixed point property, many other properties have been described. Most of these properties involve some sort of link between weak null sequences and the norm. In other words, if $x_{n} \rightarrow 0$ what can be said about the type lim sup $\left\|x-x_{n}\right\|$ where $x \in X$ ? This paper considers the effect on a Banach space when its dual has some of these properties, but first some definitions.

A bounded convex subset, $C$, of a Banach space $X$ is said to be diametral if for all $x \in C$

$$
\operatorname{diam}(C)=\sup _{y \in C}\|x-y\|,
$$

where $\sup _{y \in C}\|x-y\|$ is the radius of $C$ about $x$.

Such sets could be considered to be abnormal and hence a Banach space $X$ has normal structure if it does not contain any nontrivial bounded convex diametral subsets. Similarly $X\left(X^{*}\right)$ has weak (weak*) normal structure if it does not contain any nontrivial weak (weak*) compact convex diametral subsets. All uniformly convex spaces have normal structure. Prus [13] showed that uniformly noncreasy spaces need not have normal structure but have the fixed point property. These spaces are superreflexive and the property of uniform noncreasy is self dual. García- Falset, Llorens-Fuster and Mazcuñan-Navarro [5] have generalised the uniform noncreasy concept to r-uniformly noncreasy. If $X$ fails to have weak normal structure then there exists a sequence, $\left(x_{n}\right)$, satisfying $x_{n} \rightarrow 0$ and

$$
\lim _{n}\left\|x-x_{n}\right\|=\operatorname{diam} \overline{\operatorname{co}}\left\{x_{n}\right\}_{1}^{\infty}=1 \quad \text { for all } x \in C
$$

Since $0 \in \overline{\operatorname{co}}\left\{x_{n}\right\}_{1}^{\infty}$ we have $\lim \left\|x_{n}\right\|=1$.

Clearly weak normal structure implies the weak fixed point property. However $c_{0}$ fails to have weak normal structure but has the weak fixed point property. So weak normal structure can be considered to be a strong condition in terms of the weak fixed point property. 
Opial [10] introduced what is now called Opial's condition.

$$
\text { If } x_{n} \rightarrow 0 \text { and } x \neq 0 \text { then } \lim \sup _{n}\left\|x_{n}\right\|<\lim \sup _{n}\left\|x_{n}-x\right\| .
$$

The condition remains the same if both the limsups are replaced by lim infs. It is clear that Opial's condition implies weak normal structure.

Nonstrict Opial condition has the strict inequality replaced by ' $\leqslant$ '.

The condition, WORTH, was introduced independently by Rosenthal [14] and Sims [15]. This states that if $x_{n} \rightarrow 0$ and $x \in X$ then

$$
\lim \sup _{n}\left\|x_{n}-x\right\|=\lim \sup _{n}\left\|x_{n}+x\right\| .
$$

It is straight forward to show that WORTH implies the nonstrict Opial condition.

There are analogous definitions for weak* null sequences in $X^{*}$ which are called respectively *Opial's condition, nonstrict *Opial condition and WORTH*.

A Banach space $X$ has property $(K)$ if there exists $K \in[0,1)$ such that whenever $x_{n} \rightarrow 0,\left\|x_{n}\right\| \rightarrow 1$ and $\liminf _{n}\left\|x_{n}-x\right\| \leqslant 1$ we have $\|x\| \leqslant K$.

The condition that $\left\|x_{n}\right\| \rightarrow 1$ can be replaced by $\left\|x_{n}\right\|=1$ for all $n$.

Property $\left(K^{*}\right)$ for weak ${ }^{*}$ convergent sequences in $X^{*}$ is defined similarly.

If $K$ is not the same across $X$ but depends on the sequence $\left(x_{n}\right)$, then the condition is called property $(k)$. Clearly property $(K)$ implies property $(k)$. Similarly property $\left(K^{*}\right)$ implies property $\left(k^{*}\right)$.

Sims [16] has shown that property $(K)$ implies weak normal structure, though the proof in fact establishes the result for property $(k)$.

García-Falset [3] introduced the coefficient $R(X)$ to obtain the weak fixed point property.

$$
R(X):=\sup \left\{\lim \inf \left\|x_{n}-x\right\|: x_{n} \rightarrow 0,\left\|x_{n}\right\| \leqslant 1 \text { for all } n,\|x\| \leqslant 1\right\} .
$$

So $1 \leqslant R(X) \leqslant 2$.

Some values for $R(X)$ are, $R\left(c_{0}\right)=1$ and $R\left(L_{p}\right)=2^{1 / p}, 1 \leqslant p<\infty$.

García-Falset [3] showed that if $X$ has the nonstrict Opial condition and $R(X)<2$ then $X$ has the weak fixed point property. At about the same time Prus [12] produced a similar result. Later García-Falset [4] was able to drop the nonstrict Opial condition.

\section{RESUlts}

Now for the effect that $X^{*}$ has on $X$ if $X^{*}$ has these properties.

Proposition 1. If $X$ is a Banach space and $X^{*}$ has the nonstrict *Opial condition then $X$ has the nonstrict Opial condition. 
Proof: Assume that $X$ does not satisfy the nonstrict Opial condition then there is $\left(x_{n}\right), x$ in $X$ where $x_{n} \rightarrow 0$ and $\limsup _{n}\left\|x_{n}\right\|>\lim \sup _{n}\left\|x_{n}-x\right\|$. Passing to a subsequence we have

$$
\lim _{n}\left\|x_{n}\right\|>\lim \sup _{n}\left\|x_{n}-x\right\| \text {. }
$$

Choose $x_{n}^{*} \in S_{X^{*}}$ such that $x_{n}^{*}\left(x_{n}\right)=\left\|x_{n}\right\|$ for all $n$. Passing to a subsequence we may assume that $x_{n}^{*} \stackrel{*}{\rightarrow} x^{*} \in B_{X^{*}}$ and $\lim _{n}\left\|x_{n}^{*}-x^{*}\right\|$ exists. Nonstrict *Opial condition implies $\lim _{n}\left\|x_{n}^{*}-x^{*}\right\| \leqslant \lim _{n}\left\|x_{n}^{*}\right\|=1$. Then

$$
\begin{aligned}
\lim _{n}\left\|x_{n}-x\right\| & \geqslant \liminf _{n}\left(x_{n}^{*}-x^{*}\right)\left(x_{n}-x\right) \\
& =\lim _{n}\left\|x_{n}\right\|-x^{*}(x)+x^{*}(x) \\
& =\lim _{n}\left\|x_{n}\right\| .
\end{aligned}
$$

Contradiction.

Proposition 2. If $X$ is a Banach space, $X^{*}$ has WORTH* then $X$ has WORTH.

Proof: Assume that $X$ does not have WORTH then there exists $\left(x_{n}\right)$ in $X, x_{n} \rightarrow 0$ and $u \in X$ such that

$$
\lim \sup _{n}\left\|x_{n}+u\right\|>\lim \sup _{n}\left\|x_{n}-u\right\| .
$$

Passing to a subsequence we may assume

$$
\lim _{n}\left\|x_{n}+u\right\|>\lim _{n}\left\|x_{n}-u\right\| .
$$

Choose $x_{n}^{*} \in S_{X}$, such that $x_{n}^{*}\left(x_{n}+u\right)=\left\|x_{n}+u\right\|$ for all $n$.

Passing to a subsequence we may assume $x_{n}^{*} \stackrel{*}{\rightarrow} x^{*} \in B_{X^{*}}$. So

$$
\begin{aligned}
\lim _{n}\left\|x_{n}+u\right\| & =\lim _{n} x_{n}^{*}\left(x_{n}+u\right) \\
& =\lim _{n} x_{n}^{*}\left(x_{n}\right)+\lim _{n} x_{n}^{*}(u)-2 \lim _{n} x^{*}\left(x_{n}\right) \\
& =\lim _{n}\left(x_{n}^{*}-x^{*}\right)\left(x_{n}\right)+\lim _{n} x^{*}\left(u-x_{n}\right) \\
& =\lim _{n}\left(\left(x_{n}^{*}-x^{*}\right)-x^{*}\right)\left(x_{n}-u\right) \\
& \leqslant \lim \sup _{n}\left\|\left(x_{n}^{*}-x^{*}\right)-x^{*}\right\|\left\|x_{n}-u\right\| \\
& =\lim \sup _{n}\left\|\left(x_{n}^{*}-x^{*}\right)+x^{*}\right\|\left\|x_{n}-u\right\| \quad \text { using WORTH* } \\
& =\lim \sup _{n}\left\|x_{n}^{*}\right\|\left\|x_{n}-u\right\| \\
& =\lim \sup _{n}\left\|x_{n}-u\right\| .
\end{aligned}
$$

This contradicts the original assumption.

Note that this result is contained within the proof of Prus [12, Lemma 2.2]. Dr M. Smyth also independently obtained this result (personal communication).

Turett [18] showed that if $\varepsilon_{0}\left(X^{*}\right)<1$ then $X$ had normal structure. In fact, $X$ has uniform normal structure; see for example Prus [11]. Now if $X^{*}$ has WORTH* then the 
value of $\varepsilon_{0}\left(X^{*}\right)$ can be pushed out to be strictly less than 2 . Note that $\varepsilon_{0}\left(X^{*}\right)<2$ means that $X^{*}$ is uniformly nonsquare and is equivalent to $\lim _{\tau \rightarrow 0} \rho(\tau) / \tau<1$.

Proposition 3. Let $X$ be a Banach space where $X^{*}$ is uniformly nonsquare and has WORTH* then $X$ has normal structure.

Proof: Let $X$ be a Banach space where $X^{*}$ has WORTH* and $\varepsilon_{0}\left(X^{*}\right)<2$. Then both $X$ and $X^{*}$ are superreflexive and $X$ has WORTH. Assume $X$ does not have weak normal structure then Turett [18] showed that there exists $\left(x_{n}\right)$ in $B_{X}$ such that $x_{n} \rightarrow 0$, $\lim _{n}\left\|x_{n}\right\|=1, \operatorname{diam}\left\{x_{n}\right\}_{1}^{\infty}=1, x_{1}=0$ and $\operatorname{dist}\left(x_{n}, \operatorname{co}\left\{x_{1}, \ldots, x_{n-1}\right\}\right)>1-1 / n$.

Also, for each $n \in \mathbf{N}$, there exists $x_{n}^{*} \in S_{X}$. such that

$$
1 \geqslant x_{n}^{*}\left(x_{n}\right)-x_{n}^{*}\left(x_{j}\right)>1-1 / n \text { for } j=1, \ldots, n-1
$$

So for $j=1,1 \geqslant x_{n}^{*}\left(x_{n}\right)>1-1 / n$ for $n \geqslant 2$.

We may assume $x_{n}^{*} \rightarrow x^{*} \in B_{X^{*}}$.

Given $\varepsilon>0$ select $j_{0} \in \mathbf{N}, j_{0}>1,1 / j_{0}<\varepsilon / 2$ and $\left|x^{*}\left(x_{j_{0}}\right)\right|<\varepsilon / 4$. For $i>j_{0}$ sufficiently large

$$
\left|\left(x_{i}^{*}-x^{*}\right)\left(x_{j_{0}}\right)\right|<\varepsilon / 4,\left|x_{j_{0}}^{*}\left(x_{i}\right)\right|<\varepsilon / 2 \text { and }\left\|x_{j_{0}}-x_{i}\right\|+\varepsilon \geqslant\left\|x_{j_{0}}+x_{i}\right\| .
$$

The latter inequality is due to $X$ having WORTH. Therefore

$$
\left|x_{i}^{*}\left(x_{j_{0}}\right)\right|<\varepsilon / 2 \text {. }
$$

Then

$$
\begin{aligned}
-x_{j_{0}}^{*}\left(\frac{x_{i}-x_{j_{0}}}{\left\|x_{i}-x_{j_{0}}\right\|}\right) & \geqslant-x_{j_{0}}^{*}\left(x_{i}-x_{j_{0}}\right) \\
& =x_{j_{0}}^{*}\left(x_{j_{0}}\right)-x_{j_{0}}^{*}\left(x_{i}\right) \\
& >1-1 / j_{0}-\varepsilon / 2 \\
& >1-\varepsilon .
\end{aligned}
$$

And

$$
\begin{aligned}
x_{i}^{*}\left(\frac{x_{i}-x_{j_{0}}}{\left\|x_{i}-x_{j_{0}}\right\|}\right) & \geqslant x_{i}^{*}\left(x_{i}-x_{j_{0}}\right) \\
& =x_{i}^{*}\left(x_{i}\right)-x_{i}^{*}\left(x_{j_{0}}\right) \\
& >1-1 / i-\varepsilon / 2 \\
& >1-\varepsilon .
\end{aligned}
$$

Therefore

$$
-x_{j_{0}}^{*}, x_{i}^{*} \in S\left(B_{X^{*}}, \frac{x_{i}-x_{j_{0}}}{\left\|x_{i}-x_{j_{0}}\right\|}, \varepsilon\right)
$$


where $S\left(B_{X^{*}}, x, \delta\right)=\left\{x^{*} \in B_{X^{*}}: x^{*}(x)>1-\delta\right\}$ is the $x, \delta$-slice of $B_{X^{*}}$. Now

$$
\begin{aligned}
\left\|x_{i}^{*}-\left(-x_{j_{0}}^{*}\right)\right\| & =\left\|x_{i}^{*}+x_{j_{0}}^{*}\right\| \\
& \geqslant \frac{1}{1+\varepsilon}\left(x_{i}^{*}+x_{j_{0}}^{*}\right)\left(x_{i}+x_{j_{0}}\right) \\
& =\frac{1}{1+\varepsilon}\left(x_{i}^{*}\left(x_{i}\right)+x_{i}^{*}\left(x_{j_{0}}\right)+x_{j_{0}}^{*}\left(x_{i}\right)+x_{j_{0}}^{*}\left(x_{j_{0}}\right)\right) \\
& >\frac{1}{1+\varepsilon}\left(1-1 / i-\varepsilon / 2-\varepsilon / 2+1-1 / j_{0}\right) \\
& >\frac{2-2 \varepsilon}{1+\varepsilon} \\
& >2-4 \varepsilon .
\end{aligned}
$$

Therefore $\operatorname{diam} S\left(B_{X \cdot},\left(\left(x_{i}-x_{j_{0}}\right) /\left\|x_{i}-x_{j_{0}}\right\|\right), \varepsilon\right) \geqslant 2$.

By adapting the proof of proposition 1 of Turett $[18], \varepsilon_{0}\left(X^{*}\right)=2$, which produces a contradiction.

Takahashi and Kato [17] have shown that $X$ is uniformly nonsquare if and only if $X^{*}$ is uniformly nonsquare. This allows for an easier proof of the proposition.

From the above proof we obtain the following corollary.

Corollary 4. If $X$ is a Banach space with WORTH and $\lim _{\tau \rightarrow 0} \rho(\tau) / \tau<1$ then $X$ has normal structure.

Proposition 3 complements nicely a result of García-Falset [2] which states that if a Banach space $X$ is uniformly nonsquare and has WORTH then $X$ has the fixed point property. Sims [16] improved on this by showing that if $X$ is $\varepsilon$-inquadrate in every direction for some $\varepsilon<2$ and has WORTH then $X$ has weak normal structure.

Property $(K)$ and $R(X)<2$ are seemingly unrelated conditions but if $X^{*}$ has the nonstrict ${ }^{*}$ Opial condition then $R(X)<2$ and property $(K)$ are dual to each other.

Proposition 5. Let $X$ be a Banach space where $X^{*}$ satisfies the nonstrict *Opial condition:

(a) if $R\left(X^{*}\right)<2$ then $X$ has property $(K)$;

(b) if $X^{*}$ has property $(K *)$ then $R(X)<2$.

Proof: Recall that since $X^{*}$ has the nonstrict ${ }^{*}$ Opial condition $X$ has the nonstrict Opial condition.

(a) Let $x_{n} \rightarrow 0, \lim _{n}\left\|x_{n}\right\|=1$ and $\liminf _{n}\left\|x_{n}-x\right\| \leqslant 1$. Choose $x_{n}^{*}, y^{*} \in S_{X^{*}}$. where $x_{n}^{*}\left(x_{n}\right)=\left\|x_{n}\right\|$ for all $n$ and $y^{*}(x)=\|x\|$. Without loss of generality we may assume $x_{n}^{*} \dot{*} x^{*} \in B_{X}$. Then

$$
\begin{aligned}
\lim \sup _{n}\left(x_{n}^{*}-x^{*}-y^{*}\right)\left(x_{n}-x\right) & =1-x^{*}(x)+x^{*}(x)+y^{*}(x) \\
& =1+\|x\| .
\end{aligned}
$$


But

$$
\begin{aligned}
\lim \sup _{n}\left(x_{n}^{*}-x^{*}-y^{*}\right)\left(x_{n}-x\right) & \leqslant \lim \sup _{n}\left\|x_{n}^{*}-x^{*}-y^{*}\right\|\left\|x_{n}-x\right\| \\
& \leqslant \lim \sup _{n}\left\|x_{n}^{*}-x^{*}-y^{*}\right\| \\
& \leqslant R\left(X^{*}\right)
\end{aligned}
$$

since $\lim \sup _{n}\left\|x_{n}^{*}-x^{*}\right\| \leqslant \lim _{n}\left\|x_{n}^{*}\right\|=1$. Therefore $\|x\| \leqslant R\left(X^{*}\right)-1<1$. So $X$ has property $(K)$ with $K=R\left(X^{*}\right)-1$.

(b) Suppose $x_{n} \rightarrow 0,\left\|x_{n}\right\| \leqslant 1$ for all $n$ and $\|x\| \leqslant 1$. Select $x_{n}^{*} \in S_{X^{*}}, x_{n}^{*}\left(x_{n}+x\right)$ $=\left\|x_{n}+x\right\|$ for all $n$. We may assume $x_{n}^{*} \stackrel{*}{\rightarrow} x^{*}$ in $B_{X} \cdot$. Nonstrict ${ }^{*}$ Opial condition implies that $\lim \sup _{n}\left\|x_{n}^{*}-x^{*}\right\| \leqslant \lim _{n}\left\|x_{n}^{*}\right\|=1$. So in turn, property (K*) implies that $\left\|x^{*}\right\| \leqslant K$. Hence

$$
\begin{aligned}
\lim \sup _{n}\left\|x_{n}+x\right\| & =\lim \sup _{n} x_{n}^{*}\left(x_{n}+x\right) \\
& =\lim \sup _{n}\left(x_{n}^{*}\right)\left(x_{n}\right)+x^{*}(x) \\
& \leqslant \lim \sup _{n}\left\|x_{n}^{*}\right\|\left\|x_{n}\right\|+\left\|x^{*}\right\| \\
& =\lim \sup _{n}\left\|x_{n}\right\|+\left\|x^{*}\right\| \\
& \leqslant 1+K, \quad 0<K<1 .
\end{aligned}
$$

Therefore $R(X) \leqslant 1+K<2$.

Corollary 6 . Let $X$ be a Banach space where $X^{*}$ satisfies the nonstrict ${ }^{*}$ Opial condition. If $R\left(X^{*}\right)<2$ then $X^{*}$ has the $w^{*}$-fixed point property and $X$ has the weak fixed point property.

Combining proposition 5 with the results from Sims [16], we obtain the following corollaries.

Corollary 7. Let $X$ be a Banach space where $X^{*}$ has the nonstrict ${ }^{*}$ Opial condition. If $X^{*}$ is $\varepsilon$-URR for some $\varepsilon \in(0,1)$ then $X$ has the weak fixed point property.

Corollary 8 . Let $X$ be a Banach space where $X^{*}$ has the nonstrict ${ }^{*}$ Opial condition. If $X^{*}$ is $\varepsilon$-inquadrate in every direction for some $\varepsilon \in(0,1)$ then $X$ has the weak fixed point property.

Corollary 9. Let $X$ be a Banach space where $X^{*}$ has WORTH*. If $X^{*}$ is $\varepsilon$-inquadrate in every direction for some $\varepsilon \in(0,2)$ then $X$ has the weak fixed point property.

If $R\left(X^{*}\right)$ takes on the extreme value of 1 then some interesting things happen.

Proposition 10. If $R\left(X^{*}\right)=1$ then $R(X)=1$.

Proof: Let $x_{n} \rightarrow 0,\left\|x_{n}\right\| \leqslant 1$ for all $n$ and $\|x\| \leqslant 1$. Choose $x_{n}^{*} \in S_{X^{*}}, x_{n}^{*}\left(x_{n}\right.$ 
$+x)=\left\|x_{n}+x\right\|$ for all $n$. Without loss of generality, $x_{n}^{*} \stackrel{*}{\rightarrow} x^{*} \in B_{X} \cdot$ Then

$$
\begin{aligned}
\lim \sup _{n}\left\|x_{n}+x\right\| & =\lim \sup _{n} x_{n}^{*}\left(x_{n}+x\right) \\
& =\lim \sup _{n}\left(x_{n}^{*}-x^{*}\right)\left(x_{n}+x\right)+x^{*}(x) \\
& \leqslant \lim \sup _{n}\left\|x_{n}^{*}-x^{*}\right\|\left\|x_{n}+x\right\|+x^{*}(x) \\
& \leqslant \lim \sup _{n}\left\|x_{n}+x\right\|+x^{*}(x) \text { using } R\left(X^{*}\right)=1 .
\end{aligned}
$$

Therefore $x^{*}(x) \geqslant 0$. Similarly

$$
\begin{aligned}
\lim \sup _{n}\left\|x_{n}+x\right\| & =\lim \sup _{n} x_{n}^{*}\left(x_{n}+x\right) \\
& =\lim \sup _{n}\left(x_{n}^{*}+x^{*}\right)\left(x_{n}+x\right)-x^{*}(x) \\
& \leqslant \lim \sup _{n}\left\|x_{n}^{*}+x^{*}\right\|\left\|x_{n}+x\right\|-x^{*}(x) \\
& \leqslant \lim \sup _{n}\left\|x_{n}+x\right\|-x^{*}(x) \text { using } R\left(X^{*}\right)=1 .
\end{aligned}
$$

So $x^{*}(x) \leqslant 0$. That is, $x^{*}(x)=0=\lim _{n} x_{n}^{*}(x)$.

Hence

$$
\begin{aligned}
\lim \sup _{n}\left\|x_{n}+x\right\| & =\lim \sup _{n} x_{n}^{*}\left(x_{n}+x\right) \\
& =\lim \sup _{n} x_{n}^{*}\left(x_{n}\right) \\
& \leqslant \lim \sup _{n}\left\|x_{n}^{*}\right\|\left\|x_{n}\right\| \\
& \leqslant 1
\end{aligned}
$$

It turns out that the condition, $R\left(X^{*}\right)=1$, is very strong.

Proposition 11. If $R\left(X^{*}\right)=1$ then $X^{*}$ is not separable, $c_{0} \hookrightarrow X^{*}$ and $X$ has the Schur property.

ProOF: From proposition $5, X$ has property $(K)$ and so weak normal structure. Combining this with $R(X)=1$ leads to $X$ having the Schur property and $c_{0} 4 X$. See Dalby [1]. If $c_{0} \leftrightarrow X^{*}$ then $X^{*}$ has the weak* Schur property and so is finite dimensional. So $c_{0} \hookrightarrow X^{*}$ and $X^{*}$ is not separable. See proposition 2.e.8 and the following comments in Lindenstrauss and Tzafriri [9].

\section{REFERENCES}

[1] T. Dalby, 'Relationships between properties that imply the weak fixed point property', J. Math. Anal. Appl. 253 (2001), 578-589.

[2] J. García-Falset, 'The fixed point property in Banach spaces whose characteristic of uniform convexity is less than 2', J. Austral. Math. Soc. 54 (1993), 169-173.

[3] J. Garcia-Falset, 'Stability and fixed points for nonexpansive mappings', Houston J. Math. 20 (1994), 495-505.

[4] J. Garcia-Falset, 'The fixed point property in Banach spaces with the NUS-property', $J$. Math. Anal. Appl. 215 (1997), 532-542. 
[5] J . García-Falset, E. Llorens-Fuster and E. Mazcuñan-Navarro, 'Banach spaces which are r-uniformly noncreasy', Nonlinear Anal. (to appear).

[6] K. Goebel, 'On the structure of minimal invariant sets for nonexpansive mappings', Ann. Univ. Mariae Curie-Sklodowska Sect. A 29 (1975), 73-77.

[7] K. Goebel and W.A Kirk, Topics in metric fixed point theory, Cambridge Studies in Advanced Mathematics 28 (Cambridge University Press, Cambridge, 1990).

[8] L.A. Karlovitz, 'Existence of fixed points of nonexpansive mappings in a space without normal structure', Pacific J. Math. 66 (1976), 153-159.

[9] J. Lindenstrauss and L. Tzafriri, Classical Banach Spaces I, Sequence Spaces, Ergebnisse der Mathematik und ihrer Grenzgebiete 92 (Berlin, Heidelberg, New York, 1977).

[10] Z. Opial, 'Weak convergence of the sequence of successive approximations for nonexpansive mappings', Bull. Amer. Math. Soc. 73 (1976), 591-597.

[11] S. Prus, 'A remark on a theorem by Turett', Bull. Polish Acad. Sci. Math. 36 (1988), 225-227.

[12] S. Prus, 'Banach spaces with the uniform Opial property', Nonlinear Anal. 18 (1992), 697-704.

[13] S. Prus, 'Banach spaces which are uniformly noncreasy', Nonlinear Anal. 30 (1997), 2317-2324.

[14] H. Rosenthal, 'Some remarks concerning unconditional basic sequences', in Longhorn Notes, Texas Functional Analysis Seminar 1982-1983 (The University of Texas, Austin, TX, 1983), pp. 15-47.

[15] B. Sims, 'Orthogonality and fixed points of nonexpansive maps', Proc. Centre Math. Anal. Austral. Nat. Univ. 20 (1988), 178-186.

[16] B. Sims, 'A class of spaces with weak normal structure', Bull. Austral. Math. Soc. 49 (523-528).

[17] Y. Takahashi and M. Kato, 'Von Neumann-Jordan constant and uniformly non-square Banach spaces', Nihonkai Math. J. 9 (1998), 155-169.

[18] B. Turett, 'A dual view of a theorem of Baillon', in Nonlinear Analysis and Applications, Lecture Notes in Pure and Applied Mathematics 80 (Marcel Dekker, New York, 1982), pp. 279-286.

School of Mathematical and Physical Sciences

The University of Newcastle

New South Wales 2308

Australia
Present Address:

School of Mathematics, Statistics and Computer Science

The University of New England

New South Wales 2351

Australia 